удк 524.8

\title{
WARM DARK MATTER AND HUBBLE CONSTANT TENSIONS
}

The tensions between the values of Hubble constant obtained from the early and the late Universe could be eliminated if we use the AWDM cosmological model with dark energy, baryonic matter and warm dark matter (WDM) with characteristic velocities about $16 \%$ of the speed of light. A pressure of WDM is equal to its energy density multiplied by factor 0.009 .

Key words: dark matter, Hubble constant.

Introduction. It is known that the rate of expansion of the Universe is characterized by the time-dependant Hubble parameter $H$. Its current value is called the Hubble constant and is denoted by $H_{0}$. The value of the Hubble constant is calculated from the Hubble parameter measured in some era. This requires knowledge of both the characteristics of this era, usually its redshift $z$, and the cosmological model to relate these values.

Estimations of the Hubble constant obtained by different methods are given in [1]. Most measurements of the Hubble parameter occur at distances, which are small by cosmological standards. They have small redshifts and these measurements relate to the late Universe. However, a few measurements relate to the early Universe, more precisely to the era of recombination (redshift $z \sim 1000$ ). First of all, these are CMB data from Planck satellite [2] and data from Dark Energy Survey Year 1 clustering combined with data on weak lensing, baryon acoustic oscillations and Big Bang nucleosynthesis [3].

Some tensions between the values of Hubble constant obtained from the early and the late Universe were indicated in the recent paper [1]. I simply note that the estimation $H_{0}=67.4 \mathrm{~km} \mathrm{c}^{-1} \mathrm{Mpc}^{-1}$ obtained from observations in the era of recombination account for about $92 \%$ of the average of the estimations based on observations of not very distant objects $H_{0}=73.3 \mathrm{~km} \mathrm{c}^{-1} \mathrm{Mpc}^{-1}$. The corresponding difference is at the level of $4 \sigma-6 \sigma$, which, according to the authors, should be classified as something from a discrepancy or a problem to a crisis. They are robust to exclusion of any one method, team or source.

Naturally, the differences could be explained by measurement errors or by an influence of some poorly accounted factors. That would mean cosmology is not yet an exact science as it pretends to be. For the purposes of this work I choose to ignore possible issues with the measurement data, and consider them to be correct. I am going to show that these differences could be explained by an improvement of the standard $\Lambda$ CDM cosmological model, which does not contradict other observational data.

Theory and results. Within the framework of the flat isotropic homogeneous cosmological model the Hubble parameter change law is described by the first Friedmann equation

$$
H^{2}=\frac{8 \pi G}{3} \rho=\frac{8 \pi G}{3}\left(\rho_{m}+\rho_{d e}\right)=H_{0}^{2}\left(\frac{\rho_{m}}{\rho_{m 0}} \Omega_{m 0}+\frac{\rho_{d e}}{\rho_{d e 0}} \Omega_{d e 0}\right) .
$$

Here $\rho$ and $\Omega$ are the density and the density parameter, the subscripts $m$ and de denote matter and dark energy, the subscript 0 denotes the current value of the corresponding quantity, and $G$ is the gravitational constant. We assume that the Universe contains only matter and dark energy. This formula can also be obtained in the framework of non-relativistic cosmology [4]. I do not yet specify the dependencies of $\rho_{m}$ and $\rho_{d e}$ on $z$.

The Hubble constant value for the early Universe is calculated from the Hubble parameter obtained by processing observational data by eq. (1) for the $\Lambda$ CDM model. It assumes that the dark energy is the pure cosmological constant with constant density and both baryonic and cold dark matter (CDM) are pressureless. For this model I use the standard subscript $\Lambda$ instead of $d m$. Using the dependences of $\rho_{\Lambda}=$ const and $\rho_{m}=\rho_{m 0}(1+z)^{3}$ one get

$$
H=H_{0} \sqrt{\Omega_{\Lambda 0}+(1+z)^{3} \Omega_{m 0}} .
$$

According to the Planck satellite observations, the parameters of cosmological constant and matter density in the modern era are $\Omega_{\wedge 0}=0.68 \pm 0.02$ and $\Omega_{\mathrm{m} 0}=0.32 \pm 0.02$ [2], and their sum is fixed to 1 in the flat model. Note that these quantities are of the same order.

If the real cosmological model differed from the $\triangle \mathrm{CDM}$ one, but we used Eq. (2) instead of Eq. (1), we would get not the value $H_{0}$, but rather the product $A(z) H_{0}$

$$
H=H_{0} \sqrt{\frac{\rho_{m}}{\rho_{m 0}} \Omega_{m 0}+\frac{\rho_{d e}}{\rho_{d e 0}} \Omega_{d e 0}}=A(z) H_{0} \sqrt{\Omega_{\Lambda 0}+(1+z)^{3} \Omega_{m 0}}
$$

with the factor

$$
A(z)=\sqrt{\frac{\frac{\rho_{m}(z)}{\rho_{m 0}} \Omega_{m 0}+\frac{\rho_{d e}(z)}{\rho_{d e 0}} \Omega_{d e 0}}{\Omega_{\Lambda 0}+(1+z)^{3} \Omega_{m 0}}} .
$$

If the $\Lambda$ CDM model is correct we have $A(z)=1$. If it is not correct then $A(z)$ is almost equal to 1 for the late Universe but could differ from 1 for the early one. Let's try to explain the discrepancies in the values of the Hubble constant using Eq (4). To explain the results of the article [1] we need to provide $A(1000) \approx 0.92$. It is clear that this means to go beyond the $\Lambda$ CDM-model. 
Let's start with the letter $\Lambda$ in the name of the model and consider dark energy with variable density instead of the cosmological constant. The CDM part remains the same. We get $A(1000)<1$ if $\rho_{\text {de }}(1000)<\rho_{\text {de } 0}$, but we can not obtain $A(1000)=0.92$. Indeed,

$$
A(z)>\sqrt{\frac{(1+z)^{3} \Omega_{m 0}}{\Omega_{\Lambda 0}+(1+z)^{3} \Omega_{m 0}}}
$$

but this value is less than 1 by no more than $10^{-8}$ at $z=1000$. This is caused by the value of the $(1+z)^{3}$ factor at large $z$. It's a dead end.

Now let's try to abandon the letters CDM in the model title. This is not about the effects of electromagnetic radiation or ultrarelativistic particles and neutrinos. They exist, but their share is so small that even at $z=1000$ they do not provide the desired value of $A(z)$. So temporally forget about them and suppose that matter is composed of ordinary matter and dark matter. We know enough about baryonic matter to be sure that it can be considered as pressureless one. But we actually know just a bit about dark matter. Suppose that it has some pressure which can affect the evolution of its density. Consider the simplest form of the equation of state of dark matter

$$
p_{d m}=w \rho_{d m} c^{2},
$$

where the subscript dm means dark matter, $c$ is the speed of light and $w=$ const. Then the density of matter $\rho_{m}$ is equal to the sum of the densities of baryonic $\rho_{b}$ and dark $\rho_{d m}$ matter. They have the different dependences on $z$, namely $\rho_{b}=\rho_{b 0}(1+z)^{3}$ and $\rho_{d m}=\rho_{d m 0}(1+z)^{3+3 w}$. Substituting this into (4), we obtain the expression for $A(z)$ at large $z$ when it is possible to neglect the terms with the subscripts de and $\wedge$

$$
A(z)=\sqrt{\frac{\Omega_{m 0}}{\Omega_{b 0}+(1+z)^{3 w} \Omega_{d m 0}}} .
$$

We use the value $\Omega_{\mathrm{b} 0}=0.16 \Omega_{\mathrm{m} 0}$ based on the Planck data [2] and get the rough estimation $w=0.009$ from the condition $A(1000)=0.92$. We can make a very rough estimate of the error of the obtained $w$ value, remembering that the deviation from the case $w=0$ for the $\wedge$ CDM-model is $4 \sigma-6 \sigma$. A more accurate error estimate requires recalculation of all observational data in the framework of the proposed model, i.e. replacing a standard 6-parameter model with a 7-parameter model with an additional parameter $\mathrm{w}$ for the dark matter.

The CDM particles have characteristic velocities much less than $c$ and $w=0$, the ultrarelativistic matter like photons or neutrinos has $w=1 / 3$. The intermediate value $w=0.009$ corresponds to so-called warm dark matter (WDM). Typical velocities of the particles or quanta of this WDM are equal to the $0.16 \mathrm{c}$. I estimated it from the value of $w=0.009$ using $(6)$ and Eq. (35.8) from the book [5] for an ideal gas of identical particles of mass $m$

$$
\rho_{d m}=n m \frac{1}{\sqrt{1-\frac{v^{2}}{c^{2}}}}, p_{d m}=\frac{n m}{3} \frac{v^{2}}{\sqrt{1-\frac{v^{2}}{c^{2}}}} .
$$

Here $n$ is the number of WDM particles in a unit volume and an overline indicates averaging over all particles. Gammafactors of particles with $v \approx c / 6$ are almost 1 and one can get $\overline{v^{2}} \approx 3 w c^{2}$.

Naturally, we could also consider models in which dark matter is a mixture of CDM and WDM of different kinds. In practice this means that CDM should be added to baryonic matter in (7), and the ratio of $\Omega_{\mathrm{bo}}$ and $\Omega_{\mathrm{mo}}$ should be increased accordingly. This would lead to a corresponding increase of the value of w given by this equation. However, it wouldn't affect the principal result.

Conclusion. Thus, within the framework of the cosmological AWDM-model with $w=0.009$ for WDM we can explain the contradictions in Hubble constant values indicated in the paper [1]. However, it is still possible that tensions are caused by measurement or data processing errors.

Note that the introduction of such a small nonzero parameter $w$ is not manifested in numerous observations indicating the existence of dark matter. I mean the rotation curves of galaxies, estimates of the virial masses of galaxy clusters, gravitational lensing, the galaxy cluster mergers like the Bullet Cluster (1E 0657-56) and so on. For details see Ch. 4 in [4]. It could affect the results of modelling the growth of a large-scale structure, however.

A hypothetic WDM was introduced earlier by astrophysics to solve some problems associated with clustering on subgalactic scales and formation of halos [6]. In this article we consider cosmological aspects of WDM for explanation of tensions between the early and the late Universe. We see what the introduction of WDM provides the solution to these two different problems.

What kind of particles could form WDM? The most popular candidates are sterile neutrinos [7], gravitinos, non-thermally produced WIMPs and other particles beyond the Standard Model. WDM is often mentioned in explanations of a monochromatic signal around $3.5 \mathrm{keV}$ in the spectrum of X-ray emissions from galaxy clusters like Perseus and Centaurus observed by XMM-Newton [8,9].

The introduction of warm dark matter with the simplest equation of state (6) and $w=0.009$ explains the difference in the estimated values of the Hubble constant obtained from high- and low-redshift data and does not contradict other observational data. 
References

1. Verde L. Tensions between the early and late Universe / L. Verde, T. Treu, A. Riess // Nature Astronomy. - 2019. - Vol. 3. - P. 891-895.

2. Planck Collaboration. Planck 2018 results. VI. Cosmological parameters / Aghanim, N., Akrami, Y. et al.// 2018, arXiv:1807.06209

3. Dark Energy Survey Year 1 Results: A Precise Ho Estimate from DES Y1, BAO, and D/H Data / T.M.C. Abbott, F.B. Abdalla, J. Annis et al. // MNRAS, 2018, - Vol. 480. - P. 3879-3888.

4. Parnovsky S. How the Universe Works: Introduction to Modern Cosmology / S. Parnovsky, A. Parnowski // World Scientific Publishing Co. Pte. Ltd., 2018. - ISBN \#9789813234956.

5. Landau L.D. The classical theory of fields 1980 / L.D. Landau, E.M. Lifshitz, Butterworth-Heinemann. 1980.

6. Bode P. Halo formation in warm dark matter models / P. Bode, J.P. Ostriker, N. Turok // Astrophys. J. - 2001. - 556. - P. 93-107.

7. Dolgov A.D. Massive sterile neutrinos as warm dark matter / A.D. Dolgov, S.H. Hansen // Astroparticle Physics. - 2002. - Vol. 16. - P. 339-344.

8. Detection of an Unidentified Emission Line in the Stacked X-Ray Spectrum of Galaxy Clusters / E. Bulbul, M. Markevitch, A. Foster et al. // Astrophys. J. - 2014, - Vol. 789. - id. 13 (23 pp.)

9. Unidentified Line in X-Ray Spectra of the Andromeda Galaxy and Perseus Galaxy Cluster / A. Boyarsky, O. Ruchayskiy, D. lakubovskyi, J. Franse // Phys. Rev. Lett. - 2014. - Vol. 113. - id. 251301.

Надійшла до редколегії 11.02.20

С. Парновський, д-р фріз.-мат. наук

Київський національний університет імені Тараса Шевченка, Київ

\section{ТЕПЛА ТЕМНА МАТЕРІЯ ТА ПРОТИРІЧЧЯ У ВЕЛИЧИНІ СТАЛОЇ ХАББЛА}

Протиріччя між значеннями сталої Хаббла, отримані у ранньому та сучасному Всесвіті, можна усунути, якщо використовувати космологічну АWDM модель із темною енераією, баріонною матерією та теплою темною матерією (WDM) із характерними швидкостями порядку $16 \%$ швидкості світла. Тиск WDM дорівнює 0,009 густини її енереії.

Ключові слова: темна матерія, стала Хаббла.

С. Парновский, д-р физ.-мат. наук

Киевский национальный университет имени Тараса Шевченко, Киев

\section{ТЕПЛАЯ ТЕМНАЯ МАТЕРИЯ И ПРОТИВОРЕЧИЯ В ВЕЛИЧИНЕ ПОСТОЯННОЙ ХАББЛА}

Противоречия между значениями постоянной Хаббла, полученные в ранней и современной Вселенной можно устранить, используя космологическую АWDM модель с темной энергией, барионной материей и теплой темной материей (WDM) с характерными скоростями порядка $16 \%$ скорости света. Давление WDM равно 0,009 плотности ее энергии.

Ключевые слова: темная материя, постоянная Хаббла. 